\title{
On the stability of the universal quotient bundle restricted to congruences of low degree of $\mathbb{G}(1,3)$
}

\author{
ENRIQUE ARRONDO AND SOFÍA COBO
}

\begin{abstract}
We study the semistability of $\left.Q\right|_{S}$, the universal quotient bundle on $\mathbb{G}(1,3)$ restricted to any smooth surface $S$ (called congruence). Specifically, we deduce geometric conditions for a congruence $S$, depending on the slope of a saturated linear subsheaf of $\left.Q\right|_{S}$. Moreover, we check that the Dolgachev-Reider Conjecture (i.e. the semistability of $\left.Q\right|_{S}$ for nondegenerate congruences $S$ ) is true for all the congruences of degree less than or equal to 10. Also, when the degree of a congruence $S$ is less than or equal to 9 , we compute the highest slope reached by the linear subsheaves of $\left.Q\right|_{S}$.
\end{abstract}

Mathematics Subject Classification (2010): 14J60 (primary); 14M07, 14M15 (secondary).

\section{Introduction}

The main numerical invariant of a congruence, i.e. a smooth irreducible surface of the Grassmann variety $\mathbb{G}(1,3)$ of lines in $\mathbb{P}^{3}$, is its bidegree $(a, b)$. Regarding a congruence as a two-dimensional family of lines in $\mathbb{P}^{3}$, the order $a$ is defined to be the number of lines of the family passing through a general point of $\mathbb{P}^{3}$; analogously, the class $b$ is the number of lines of the family contained in a general plane of $\mathbb{P}^{3}$. An interesting problem that arises in a natural way is the following: given two integers $a$ and $b$, does there exist a congruence of bidegree $(a, b)$ ?

About this question, the best known result is the bound $a \leq O\left(b^{\frac{4}{3}}\right)$ for every congruence of bidegree $(a, b)$, given by Gross in [8] (although there are not known examples for which $|a-b|>O\left(b^{3 / 4}\right)$ ). A new approach was introduced by Dolgachev and Reider by means of vector bundles. More precisely, they stated in [7] the following conjecture:

This paper has been written in the framework of the research projects MTM2006-04785 (funded by the Spanish Ministry of Education) and CCG07-UCM/ESP-3026 (funded by University Complutense and the regional government of Madrid).

Received February 11, 2009; accepted in revised form July 24, 2009. 
Conjecture DR. Let $S$ be a non-degenerate congruence, i.e. not lying on a hyperplane section of $\mathbb{G}(1,3)$. Then the restriction to $S$ of the rank-two universal quotient bundle of $\mathbb{G}(1,3)$ is semistable.

The main interest of this conjecture lies in the fact that, if true, Bogomolov's theorem would imply $a \leq 3 b$ for all congruences of bidegree $(a, b)$, except for the congruence of lines passing through one fixed point of $\mathbb{P}^{3}$. Dually, $b \leq 3 a$ for all congruences of bidegree $(a, b)$, except for the congruence of lines contained in a fixed plane.

Few results are known about the stability of the restriction of $Q$ to congruences. For example, the congruences $S$ of $\mathbb{G}(1,3)$ for which $\left.Q\right|_{S}$ splits were classified in [3], while those for which $\left.Q\right|_{S}$ is not simple were classified in [4], showing that they are precisely those for which $\left.Q\right|_{S}$ splits.

Regarding the concrete problem of the study of the stability of the restriction of $Q$ to a congruence of $\mathbb{G}(1,3)$, Gross proves in [8] (see [3] for another proof) that, given a congruence $S$ of bidegree $(a, b)$ and $b \geq a$, then $\left.Q\right|_{S}$ is semistable.

In this paper, we start by using some implicit information in the proof of Gross result to obtain geometric properties of a congruence $S$, depending on the slope of some linear subsheaf of $\left.Q\right|_{S}$ (see Theorem 2.1). Although this result does not enable us to answer Conjecture DR, we are able to deduce from it a certain estimation of the highest slope reached by the linear subsheaves of $\left.Q\right|_{S}$. Since this estimation has significance only when $|a-b|$ is small, we check the validity of Conjecture DR for all the congruences of degree less than or equal to 10 .

The main observation is that the stability of the restriction of $Q$ to a congruence, or the highest slope reached by its linear subsheaves, can vary within the same family of congruences. An example of this situation is the case of degenerate congruences of even degree: for a general congruence of the family of such congruences the restriction of $Q$ is stable, but it is semistable (and not stable) for a few special cases. In order to speak of the general or the special congruence within the same family it is necessary that the Hilbert scheme parameterizing this family is irreducible. Since this irreducibility is only known for congruences of degree less than or equal to 9 (see [3]), the study of the highest slope for congruences of higher degree is beyond the scope of this paper. However, we determine the stability of $\left.Q\right|_{S}$ for every type of congruence $S$ with degree less than or equal to 10 and, when $S$ is general and of degree less than or equal to 9 , we compute the highest slope reached by the linear subsheaves of $\left.Q\right|_{S}$. When possible, we also compute this highest slope for some special cases.

Our results lead us to formulate the following:

Conjecture 0.1. For every irreducible component of the Hilbert scheme of nondegenerate congruences, the restriction of $Q$ to the general congruence is semistable.

Observe that, although the above conjecture is weaker than Conjecture DR, however both imply the same relation $a \leq 3 b$ for all the congruences of bidegree $(a, b)$ (except for the congruence of lines through a point), because the bidegree is constant in each component of the Hilbert scheme. 
The structure of this paper is as follows. We start in Section 1 by recalling several well-known facts about congruences of lines in $\mathbb{P}^{3}$. Moreover, we recall the list of all types of congruences of $\mathbb{G}(1,3)$ of degree less than or equal to 9. In Section 2 we present first the main general result (Theorem 2.1) about the stability of the restriction of $Q$ that we prove in this paper. Moreover, we prove that Conjecture DR is true for all the congruences up to degree 9, and determine, for the general congruence $S$ of each type, the highest slope reached by the linear subsheaves of $\left.Q\right|_{S}$ (see Theorem 2.3). In this section we only prove Theorem 2.1, devoting Section 3 to the (long) proof of Theorem 2.3. In order to make this section more readable, we divide it into smaller subsections, each of them collecting congruences sharing certain geometric properties. In a final appendix we present in a table all the partial results we have got about the congruences of degree 10, in order to illustrate the difficulties appearing in higher degree.

\section{Preliminaries}

Fix a four-dimensional vector space $V$ over the complex field $\mathbb{C}$ and denote by $\mathbb{P}^{3}$ the projective space $\mathbb{P}(V)$ of one-dimensional quotients of $V$. We denote by $\mathbb{G}(1,3)$ the Grassmann variety of lines of $\mathbb{P}^{3}$. We will frequently regard $\mathbb{G}(1,3)$ as a smooth quadric in $\mathbb{P}^{5}$ via the Plücker embedding.

We denote by $Q$ and $\mathcal{S}$, respectively, the universal quotient bundle and the universal subbundle, defined in the universal exact sequence:

$$
0 \longrightarrow \mathcal{S}^{*} \longrightarrow H^{0}\left(\mathcal{O}_{\mathbb{P}^{3}}(1)\right) \otimes \mathcal{O}_{\mathbb{G}(1,3)} \longrightarrow Q \longrightarrow 0 .
$$

Taking cohomology in the above sequence, we obtain

$$
H^{0}(Q)=H^{0}\left(\mathcal{O}_{\mathbb{P}^{3}}(1)\right)=V,
$$

and, more precisely, the zero locus of any nonzero section of $Q$ is the set of lines contained in the plane of $\mathbb{P}^{3}$ defined by the corresponding element of $V$ (such a set of lines is called a $\beta$-plane). Dually, any nonzero section of $\mathcal{S}$ vanishes on the set of lines passing through a given point of $\mathbb{P}^{3}$ (such a set is called an $\alpha$-plane).

Definition 1.1. A complex is a hypersurface of $\mathbb{G}(1,3)$, which is necessarily the complete intersection of $\mathbb{G}(1,3)$ with a hypersurface of $\mathbb{P}^{5}$. The degree of the latter is called the degree of the complex. Up to projective equivalence, there are only two types of linear complexes, depending on whether the corresponding hyperplane of $\mathbb{P}^{5}$ is tangent or not to $\mathbb{G}(1,3)$. In the first case, we obtain a special linear complex, which is the set of all lines of $\mathbb{P}^{3}$ meeting a fixed line.

Definition 1.2. By congruence, we mean a smooth irreducible surface in the Grassmannian $\mathbb{G}(1,3)$. Since there is a natural isomorphism between $\mathbb{G}\left(1, \mathbb{P}^{3}\right)$ and $\mathbb{G}\left(1, \mathbb{P}^{3 *}\right)$, it makes sense to speak about the dual congruence of a given congruence. 
The number $a$ of lines of a congruence passing through a general point of $\mathbb{P}^{3}$ is called order of the congruence. Dually, the number $b$ of lines of a congruence contained in a general plane of $\mathbb{P}^{3}$ is called class of the congruence (obviously, $\left.b=c_{2}\left(\left.Q\right|_{S}\right)\right)$. The pair $(a, b)$ is called the bidegree of the congruence. Clearly, the dual congruence of a congruence of bidegree $(a, b)$ has bidegree $(b, a)$. Finally, it is easy to check that the degree of a congruence of bidegree $(a, b)$ as a surface of $\mathbb{G}(1,3) \subset \mathbb{P}^{5}$ is $a+b$. We will also write $\pi$ for the sectional genus of a congruence as a surface in $\mathbb{P}^{5}$.

We say that a congruence has a fundamental curve if there is a curve in $\mathbb{P}^{3}$ meeting all the lines of the congruence. Observe that a congruence is degenerate (i.e. contained in a hyperplane of $\mathbb{P}^{5}$ ) if and only if it is contained in a linear complex. Moreover, the congruence has a fundamental line if and only if it is contained in a special linear complex.

Definition 1.3. Given a congruence $S$, by stability (respectively semistability) of $\left.Q\right|_{S}$ we will mean $\mu$-stability (respectively $\mu$-semistability) with respect to the hyperplane section $H$ of $S$ as a surface in $\mathbb{P}^{5}$. In other words, $\left.Q\right|_{S}$ is stable if, for any subsheaf $\mathcal{O}_{S}(D)$ of $\left.Q\right|_{S}$, we have $\mu(D)<\mu\left(\left.Q\right|_{S}\right.$ ), i.e. $D H<\frac{a+b}{2}$ (for semistability just replace $<$ with $\leq$ ).

Remark 1.4. Notice that it suffices to check the stability condition for saturated subsheaves $\mathcal{O}_{S}(D)$ of $\left.Q\right|_{S}$, i.e. such that the corresponding section of $\left.Q\right|_{S}(-D)$ vanishes only at a finite number of points $Z$. In this case there exists an exact sequence

$$
\left.0 \longrightarrow \mathcal{O}_{S}(D) \longrightarrow Q\right|_{S} \longrightarrow \mathcal{J}_{Z}(H-D) \longrightarrow 0
$$

and $\operatorname{deg} Z=c_{2}\left(\left.Q\right|_{S}(-D)\right)=c_{2}\left(\left.Q\right|_{S}\right)-c_{1}\left(\left.Q\right|_{S}\right) D+D^{2}=b-D H+D^{2}$. It is easy to prove that, if $\mu(D)$ is the highest slope of all possible subsheaves of $\left.Q\right|_{S}$, then $\mathcal{O}_{S}(D)$ is saturated. From now on, we will say that such $\mu(D)$ is the highest slope for $S$.

Now we present in Table 1.1 all the congruences of degree less than or equal to 9, collected by Arrondo and Sols in [3].

We omit in each case the dual congruence since, apart from the bidegree, the rest of the invariants and the hyperplane section are identical. In case that a congruence is degenerate, we specify it in the table. With respect to the notation, $\widetilde{X}\left(x_{1}, \ldots, x_{r}\right)$ denotes the blow-up of a surface $X$ along the points $x_{1}, \ldots, x_{r}$ with exceptional divisors $E_{1}, \ldots, E_{r}$. For ruled surfaces we keep the notation of [10, V, Section 2] (this works also for conic bundles, which are blow-ups of ruled surfaces). Finally, by c.i. $\left(d_{1}, d_{2}\right)$ we mean the complete intersection in $\mathbb{G}(1,3)$ of two complexes of degree $d_{1}$ and $d_{2}$.

We recall that, as proved in [3], the Hilbert scheme of each of the above types of congruences is irreducible. 

$(a, b) \pi \quad$ Description
Embedding in $\mathbb{P}^{5}$

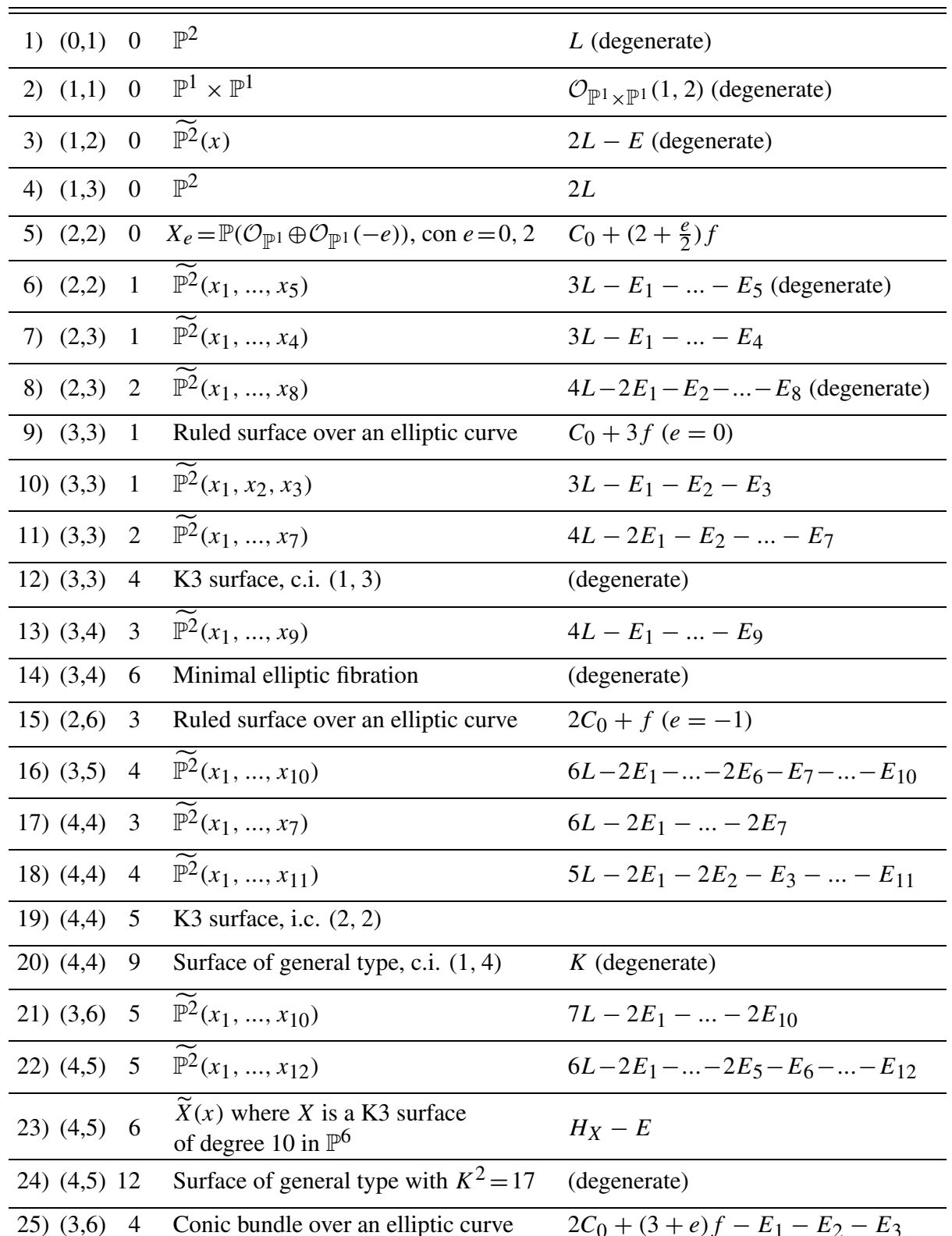

Table 1.1. Congruences of degree $\leq 9$. 
Let us collect some useful lemmas and well-known results that we will need later on:

Lemma 1.5. For any congruence $S$, the morphism $H^{0}(Q) \stackrel{\varphi}{\longrightarrow} H^{0}\left(\left.Q\right|_{S}\right)$ is an isomorphism, except when $S$ is of one of the following types:

i) a $\beta$-plane, dual of the congruence of type 1$)$,

ii) the congruence $(2,1)$, dual of the congruence of type 3$)$,

iii) the congruence $(2,2)$ of type 5$)$,

iv) the Veronese surface $(3,1)$, dual of the congruence of type 4),

v) the congruence $(3,2)$, dual of the congruence of type 7$)$,

vi) the congruence $(3,3)$ of type 10$)$.

Proof. The morphism $\varphi$ is not injective if and only if there is a section $s$ of $Q$ that is zero when restricted to $S$. Since the zero locus of a section of $Q$ is a $\beta$-plane, it follows that $\varphi$ is not injective if and only if $S$ is a $\beta$-plane. Hence we can assume that $\varphi$ is injective.

Suppose now that $\varphi$ is not an epimorphism. Then necessarily $h^{0}\left(\left.Q\right|_{S}\right) \geq 5$ and, by [3, Theorem 5.1], $S$ is of one of the types ii),..,vi).

Lemma 1.6. Let $S$ be a congruence having a fundamental curve. Then, one of the following holds:

(i) $C$ is a line, so that $S$ is degenerate.

(ii) $C$ is a plane conic, and $S$ is either dual of a congruence of type 3) or a congruence of type 5).

(iii) $C$ is a plane cubic, and $S$ is a particular case of congruence of type 25).

(iv) $C$ is a twisted cubic, and $S$ is a congruence of type 4).

Proof. See [2].

Remark 1.7. We recall from [3] that, if $S$ is a degenerate congruence, one of the following holds:

(i) $a=b$, and $S$ is the complete intersection of a linear complex and a complex of degree $a$. In particular, when $a>1, S$ has a fundamental line if and only if the linear complex is special. If $a=1$, i.e. $S$ is of type 2), the congruence is the complete intersection of two linear complexes; therefore $S$ is always contained in a special linear complex, which implies that it has a fundamental line.

(ii) $a=b-1$ and $S$ has a fundamental line.

(iii) $b=a-1$ and $S$ is dual of a congruence as in ii). In particular, it has a fundamental line. 
An easy property that we will often use is the following:

Remark 1.8. Consider a plane curve $C$ in $\mathbb{G}(1,3)$ of degree greater than or equal to 3 , and let $\Pi$ be the plane of $\mathbb{P}^{5}$ containing $C$. Then every line in $\Pi$ is contained in $\mathbb{G}(1,3)$, hence $\Pi$ is contained in $\mathbb{G}(1,3)$. Since the only planes of $\mathbb{P}^{5}$ contained in $\mathbb{G}(1,3)$ are the $\alpha$-planes and the $\beta$-planes, we get that $C$ is contained either in an $\alpha$-plane or in a $\beta$-plane.

\section{General results}

We state in this section the two main results of this paper, the second one (which we will prove in the next section) showing in particular that Conjecture DR is true for all congruences with degree less than or equal to 9. In the first result, using ideas that were implicit in [8], we determine a bound for the slope $\mu(D)$ of the subsheaves $\left.\mathcal{O}_{S}(D) \subset Q\right|_{s}$. Moreover, we show that, when $\mu(D)$ is very close to this bound, $S$ must be of a very special type. This allows us to deduce results on the stability of $\left.Q\right|_{S}$ :

Theorem 2.1. Let $S$ be a congruence in $\mathbb{G}(1,3)$ of bidegree $(a, b)$. Let us suppose that we have an exact sequence like in (1.2). Then:

i) $\mu(D) \leq a$.

ii) If $\mu(D)=a$, then $S$ has a fundamental curve.

iii) If $\mu(D)=a-1$, then $S$ is rational. Moreover, if $h^{0}\left(\left.Q\right|_{S}\right)=4$, then $h^{0}(D)=1$.

iv) If $\mu(D)=a-2$, then either $S$ is rational or $h^{0}(D)=1$.

v) If $\mu(D)=a-3$, then either $S$ is birational to $\mathbb{P}^{2}$ or to an elliptic ruled surface, or $h^{0}(D)=1$.

In particular,

$\left.i^{\prime}\right)$ if $a \leq b$ (respectively $\left.a<b\right)$, then $\left.Q\right|_{S}$ is semistable (respectively stable).

$\left.\mathrm{ii}^{\prime}\right)$ if $S$ has no fundamental curve and $a \leq b+2$ (respectively $a<b+2$ ), then $\left.Q\right|_{S}$ is semistable (respectively stable).

iii') If $S$ is not rational and has no fundamental curve, and if $a \leq b+4$ (respectively $a<b+4$ ), then $\left.Q\right|_{S}$ is semistable (respectively stable).

Proof. Let $\left.H^{0}\left(\mathcal{O}_{\mathbb{P}^{3}}(1)\right) \otimes \mathcal{O}_{S} \rightarrow Q\right|_{S} \rightarrow \mathcal{J}_{Z}(H-D)$ be the composition of the epimorphism of the universal sequence (1.1) restricted to $S$ and the epimorphism of (1.2). Let $\pi: \widetilde{S} \rightarrow S$ be the blow-up of $S$ along $Z$ and set $E=\pi^{-1}(Z)$ (hence $E^{2}=-\operatorname{deg} Z$ ). The pull-back by $\pi$ of such composition is an epimorphism $H^{0}\left(\mathcal{O}_{\mathbb{P}^{3}}(1)\right) \otimes \mathcal{O}_{\tilde{S}} \rightarrow \mathcal{O}_{\widetilde{S}}(H-D-E)$, which defines a map $\psi: \widetilde{S} \longrightarrow \mathbb{P}^{3}$.

Since $\mathcal{O}_{\widetilde{S}}(H-D-E)$ is generated by its global sections, $(H-D-E)^{2} \geq 0$ which, using $\operatorname{deg} Z=b-D H+D^{2}$ (Remark 1.4), becomes $D H \leq a$, proving i). 
If $D H=a$, then $(H-D-E)^{2}=0$, so that $\psi(\widetilde{S})$ must be either a curve or a point. In the first case, $\psi(\widetilde{S})$ is a fundamental curve for $S$. In the second case $S$ is obviously an $\alpha$-plane; therefore every line of $S$ is fundamental. This proves ii).

If $D H_{\widetilde{S}}=a-1$ then $(H-D-E)_{\widetilde{S}}^{2}=1$, then $\operatorname{deg} \psi=\operatorname{deg} \psi(\widetilde{S})=1$. Therefore $\widetilde{S}$ is birational to the plane $\psi(\widetilde{S})$, so that $S$ is rational. If, moreover, $h^{0}\left(\left.Q\right|_{S}\right)=4$, we have (see Lemma 1.5) an isomorphism $H^{0}\left(\mathcal{O}_{\mathbb{P}^{3}}(1)\right) \cong H^{0}\left(\left.Q\right|_{S}\right)$. Since the image of the map $H^{0}\left(\mathcal{O}_{\mathbb{P}^{3}}(1)\right) \rightarrow H^{0}\left(\mathcal{J}_{Z}(H-D)\right)$ has dimension three (because the image of $\psi$ is a plane), it follows that its kernel, which is $H^{0}(D)$, has dimension one, completing the proof of iii).

If now $D H=a-2$, then either $\operatorname{deg} \psi=1, \operatorname{deg} \psi(\widetilde{S})=2$ or $\operatorname{deg} \psi=$ $2, \operatorname{deg} \psi(\widetilde{S})=1$. In the first case, $\widetilde{S}$ is birational to a quadric (hence rational), and so is $S$. In the second case, $\psi(\widetilde{S})$ is a plane; moreover, if $h^{0}\left(\left.Q\right|_{S}\right)=4$, one can prove exactly as in iii) that $h^{0}(D)=1$. Since, if $h^{0}\left(\left.Q\right|_{S}\right) \neq 4$, the congruence $S$ is rational by Lemma 1.5, we have proved iv).

Finally, if $D H=a-3$, we distinguish two possibilities. If $\operatorname{deg} \psi=1$ and $\operatorname{deg} \psi(\widetilde{S})=3$, then $\widetilde{S}$ is birational to a surface of degree three in $\mathbb{P}^{3}$, which is necessarily rational or a cone over an elliptic curve. Therefore, $S$ is either rational or birational to an elliptic ruled surface. If instead $\operatorname{deg} \psi=3$ and $\operatorname{deg} \psi(\widetilde{S})=1$, then $\psi(\widetilde{S})$ must be a plane. Thus, in the same way as in iii) or iv), $h^{0}\left(\left.Q\right|_{S}\right)=4$ implies $h^{0}(D)=1$, while if $h^{0}\left(\left.Q\right|_{S}\right) \neq 4$, then $S$ is rational.

This proves v).

Parts $\left.\left.\mathrm{i}^{\prime}\right), \mathrm{ii}^{\prime}\right)$, iii'), follow readily from i), ii), iii), since, as we observed in Remark 1.4, a subsheaf $\mathcal{O}_{S}(D)$ of $\left.Q\right|_{S}$ with the highest possible slope is saturated, so that we have an exact sequence as in (1.2).

Remark 2.2. Observe that, whenever there exists a curve $D \subset S$ contained in a $\beta$ plane, there exists a subsheaf $\mathcal{O}_{S}(D)$ of $\left.Q\right|_{S}$ such that $h^{0}(D)>0$. And, by Lemma 1.5 , both statements are equivalent if $h^{0}\left(\left.Q\right|_{S}\right)=4$.

The following theorem (which we will prove in the next section) contains all the results we have got on the stability of $Q$ restricted a congruence $S$ of degree less than or equal to 9 . We will present such results in a table, in which, for each type of congruence, we keep the same numeration we have introduced in Section 1. The only difference is that now, when $a \neq b$, we have to distinguish between one type of congruence and its dual, since the stability behavior of the restriction of $Q$ is different. We will thus use an asterisk to denote the dual type of a congruence. In the column $\mu_{\max }$ we will write the highest slope reached by the linear subsheaves of $\left.Q\right|_{S}$, for the general congruence $S$ of each type. In the cases in which we know that there exist special congruences with slope greater than the general $\mu_{\max }$, we specify this special $\mu_{\max }$ in the last column. This information does not intend to be exhaustive at all: for example, in case 12), there might be special congruences without fundamental line and $\mu_{\max }=2$ or 1 .

Theorem 2.3. Let $S$ be a congruence in $\mathbb{G}(1,3)$ with degree less than or equal to 9. Then the stability properties of $\left.Q\right|_{S}$ are described in Table 2.1 . 
$(a, b) \quad \pi \quad \mu\left(\left.Q\right|_{S}\right) \quad \mu_{\max } \quad$ Stability $\left.Q\right|_{S}$ general $\quad \mu_{\max }$ special

\begin{tabular}{|c|c|c|c|c|c|c|}
\hline 1) & $(0,1)$ & 0 & $1 / 2$ & 0 & Stable & There is no one \\
\hline 1$)^{*}$ & $(1,0)$ & 0 & $1 / 2$ & 1 & Unstable & There is no one \\
\hline 2) & $(1,1)$ & 0 & 1 & 1 & Semistable non stable & There is no one \\
\hline 3) & $(1,2)$ & 0 & $3 / 2$ & 1 & Stable & There is no one \\
\hline 3$)^{*}$ & $(2,1)$ & 0 & $3 / 2$ & 2 & Unstable & There is no one \\
\hline 4) & $(1,3)$ & 0 & 2 & 0 & Stable & There is no one \\
\hline 4$)^{*}$ & $(3,1)$ & 0 & 2 & 2 & Semistable & There is no one \\
\hline 5) & $(2,2)$ & 0 & 2 & 2 & Semistable non stable & There is no one \\
\hline 6) & $(2,2)$ & 1 & 2 & 1 & Stable & 2 iff fundamental line \\
\hline 7) & $(2,3)$ & 1 & $5 / 2$ & 1 & Stable & There is no one \\
\hline 7$)^{*}$ & $(3,2)$ & 1 & $5 / 2$ & 2 & Stable & There is no one \\
\hline 8) & $(2,3)$ & 2 & $5 / 2$ & 2 & Stable & There is no one \\
\hline 8$)^{*}$ & $(3,2)$ & 2 & $5 / 2$ & 3 & Unstable & There is no one \\
\hline 9) & $(3,3)$ & 1 & 3 & 3 & Semistable non stable & There is no one \\
\hline 10) & $(3,3)$ & 1 & 3 & 2 & Stable & There is no one \\
\hline 11) & $(3,3)$ & 2 & 3 & 1 & Stable & 2 if conic in $\beta$-plane \\
\hline 12) & $(3,3)$ & 4 & 3 & 0 & Stable & 3 iff fundamental line \\
\hline 13) & $(3,4)$ & 3 & $7 / 2$ & 1 & Stable & $?$ \\
\hline 13$)^{*}$ & $(4,3)$ & 3 & $7 / 2$ & 3 & Stable & There is no one \\
\hline 14) & $(3,4)$ & 6 & $7 / 2$ & 3 & Stable & There is no one \\
\hline 14$)^{*}$ & $(4,3)$ & 6 & $7 / 2$ & 4 & Unstable & There is no one \\
\hline 15$)$ & $(2,6)$ & 3 & 4 & 0 & Stable & There is no one \\
\hline 15$)^{*}$ & $(6,2)$ & 3 & 4 & 3 & Stable & There is no one \\
\hline 16) & $(3,5)$ & 4 & 4 & 1 & Stable & $?$ \\
\hline 16$)^{*}$ & $(5,3)$ & 4 & 4 & 3 & Stable & $?$ \\
\hline 17) & $(4,4)$ & 3 & 4 & 0 & Stable & $?$ \\
\hline 18) & $(4,4)$ & 4 & 4 & 2 & Stable & $?$ \\
\hline 19) & $(4,4)$ & 5 & 4 & 0 & Stable & $?$ \\
\hline 20) & $(4,4)$ & 9 & 4 & 0 & Stable & 4 iff fundamental line \\
\hline 21) & $(3,6)$ & 5 & $9 / 2$ & 0 & Stable & $?$ \\
\hline 21$)^{*}$ & $(6,3)$ & 5 & $9 / 2$ & 3 & Stable & There is no one \\
\hline 22) & $(4,5)$ & 5 & $9 / 2$ & 1 & Stable & $?$ \\
\hline 22$)^{*}$ & $(5,4)$ & 5 & $9 / 2$ & 2 & Stable & $?$ \\
\hline 23) & $(4,5)$ & 6 & $9 / 2$ & 1 & Stable & $?$ \\
\hline 23$)^{*}$ & $(5,4)$ & 6 & $9 / 2$ & 1 & Stable & $?$ \\
\hline 24) & $(4,5)$ & 12 & $9 / 2$ & 4 & Stable & There is no one \\
\hline 24$)^{*}$ & $(5,4)$ & 12 & $9 / 2$ & 5 & Unstable & There is no one \\
\hline 25$)$ & $(3,6)$ & 4 & $9 / 2$ & 1 & Stable & 3 if fundamental curve \\
\hline 25$)^{*}$ & $(6,3)$ & 4 & $9 / 2$ & 3 & Stable & There is no one \\
\hline
\end{tabular}

Table 2.1. Stability properties for congruences of degree $\leq 9$. 


\section{Proof of Theorem 2.3}

We prove briefly every case, giving only more detailed proofs for those congruences presenting difficulties or interesting peculiarities (the complete details can be found in [5]). Moreover, we subdivide the proof in six subsections by putting together congruences with similar properties.

\subsection{Immediate cases}

Case 1). In this case, it is known that $\left.Q\right|_{S}=\Omega_{\mathbb{P}^{2}}$ (2), which is stable. Therefore the highest slope is 0 .

Case 1)*. Since $\left.Q\right|_{S}=\mathcal{O}_{\mathbb{P}^{2}} \oplus \mathcal{O}_{\mathbb{P}^{2}}(1)$, we have that $\mathcal{O}_{\mathbb{P}^{2}}(1)$ is a destabilizing subsheaf of $\left.Q\right|_{S}$. Hence $\left.Q\right|_{S}$ is unstable and the highest slope for $S$ is 1 .

Case 4). Since $b>a$, it follows from Theorem 2.1 that $\left.Q\right|_{S}$ is stable and also $\mu(D) \leq 1$ for all $\left.\mathcal{O}_{S}(D) \subset Q\right|_{S}$. Since $S=\mathbb{P}^{2}$ with hyperplane section $H=2 L$, $\mu_{\text {max }}$ is even, so it is zero.

Case 4)*. We know in this case that $\left.Q\right|_{S}=\mathcal{O}_{\mathbb{P}^{2}}(1) \oplus \mathcal{O}_{\mathbb{P}^{2}}(1)$, hence $\left.Q\right|_{S}$ is not stable. This implies that $\mathcal{O}_{\mathbb{P}^{2}}(1)$ is a subsheaf of $\left.Q\right|_{S}$, which gives $\mu_{\max }=2$.

\subsection{Congruences with plane fundamental curve}

According to Lemma 1.6 and Remark 1.7, the families of congruences for which the general one has a plane fundamental curve are cases 2$\left.\left.\left.\left.), 3), 3)^{*}, 5\right), 8\right), 8\right)^{*}, 9\right)$, 14), 14)*, 24), 24)*.

First of all, we prove a result for congruences with plane fundamental curve, which will allow us to calculate the highest slope and to decide whether $\left.Q\right|_{S}$ is stable or not:

Proposition 3.1. Let $S$ be a congruence of bidegree $(a, b)$. If $S$ has a plane fundamental curve (in particular, if $S$ has a fundamental line) then there exists a linear subsheaf of $\left.Q\right|_{S}$ with slope equal to a. Therefore $\mu_{\max }=a$.

Proof. By Remark 2.2, it will be enough to find a curve $D \subset S$ of degree $a$ contained in a $\beta$-plane (the last statement of the proposition follows from Theorem 2.1 i)). Let $C$ be a fundamental curve of $S$ contained in a plane $\Pi \subset \mathbb{P}^{3}$, and let $p$ be a general point of $\Pi$. Since $C$ is a fundamental curve for $S$, all the lines of $S$ passing through $p$ meet $C$, and are thus contained in the plane $\Pi$. Now, since the order of $S$ is $a$, there are exactly $a$ lines of $S$ passing through $p$. Hence the one-dimensional part of the set of lines of $S$ contained in $\Pi$ is a curve of degree $a$, which completes the proof.

We now apply Proposition 3.1 to each case mentioned above:

Case 2). Any congruence of this type has a fundamental line, so $\mu_{\max }=1$, and $\left.Q\right|_{S}$ is semistable but not stable. 
Cases 3), 8), 14), 24). They all are degenerate congruences of bidegree $(a, a+1)$ with fundamental line, so $\mu_{\max }=a$ and $\left.Q\right|_{S}$ is stable.

Cases 3$\left.\left.\left.)^{*}, \mathbf{8}\right)^{*}, \mathbf{1 4}\right)^{*}, \mathbf{2 4}\right)^{*}$. They are degenerate congruences of bidegree $(a, a-1)$ with fundamental line, so that $\mu_{\max }=a$ and $\left.Q\right|_{S}$ is unstable.

Case 5). Any congruence of this type has a plane fundamental conic, so that $\mu_{\max }=$ 2 , and $\left.Q\right|_{S}$ is semistable but not stable. We could also include here the congruence of type 3$)^{*}$, since it also has a plane fundamental conic, but it was considered in the previous case, because it has a fundamental line too.

Case 9). Congruences of this type have a plane fundamental cubic, and hence $\mu_{\max }=3$, and $\left.Q\right|_{S}$ is semistable but not stable.

\subsection{General complete intersections}

In order to study the stability of $Q$ restricted to congruences which are general complete intersections, we need to prove the following:

Proposition 3.2. Let $S$ be a general complete intersection in $\mathbb{G}(1,3)$ of two complex of degrees $d_{1}$ and $d_{2}$, with $\left(d_{1}, d_{2}\right) \neq(1,1),(1,2),(2,1)$. Then every invertible subsheaf of $\left.Q\right|_{S}$ has the form $\mathcal{O}_{S}(d H)$, with $d \leq 0$. In particular, $\left.Q\right|_{S}$ is stable and $\mu_{\max }=0$.

Proof. First, we use a classical theorem of Noether and Lefschetz (see [6] for a modern proof), which claims that, if $S$ is a general complete intersection surface in $\mathbb{P}^{n}$, then $\operatorname{Pic}(S)$ is generated by the hyperplane section except if $S$ is one of the following cases:

a) a quadric surface in $\mathbb{P}^{3}$,

b) a cubic surface in $\mathbb{P}^{3}$,

c) a complete intersection in $\mathbb{P}^{4}$ of two quadric hypersurfaces.

Note that $\left(d_{1}, d_{2}\right)=(1,1)$ corresponds to case $\left.a\right)$, that case $b$ ) cannot happen (since $\operatorname{deg} S$ is even) and that $\left(d_{1}, d_{2}\right)=(1,2),(2,1)$ corresponds to case $\left.c\right)$. Therefore, we can assert in our situation that $\operatorname{Pic}(S)$ is generated by the hyperplane section. Hence every linear subsheaf on $S$ takes the form $\mathcal{O}_{S}(d H)$. Let us suppose, by contradiction, that $d \geq 1$. Then $\left.\mathcal{O}_{S}(H) \subset \mathcal{O}_{S}(d H) \subset Q\right|_{S}$, which implies that $h^{0}\left(\left.Q\right|_{S} ^{*}\right)=h^{0}\left(\left.Q\right|_{S}(-H)\right) \neq 0$. Since $\left.Q\right|_{S}$ is generated by its global sections, it splits with $\mathcal{O}_{S}$ as a direct summand. We thus have a surjective morphism $\left.Q\right|_{S} \rightarrow \mathcal{O}_{S}$, which implies that $S$ is an $\alpha$-plane. But an $\alpha$-plane is not a complete intersection in $\mathbb{G}(1,3)$, so that we get the wanted contradiction.

Cases 12), 19), 20), 23), 23)*. The general congruence of each one of these types is a general complete intersection in $\mathbb{G}(1,3)$. Applying Proposition 3.2 we know that, for the general congruence of each of the above types, the restricted universal bundle is stable and $\mu_{\max }=0$. 
Remark 3.3. Among the cases above, we have types 12) and 20), which are degenerate congruences of bidegree $(a, a)$. By Remark 1.7, we know that there are special congruences of these types having a fundamental line. By Theorem 2.1 iii), in these special cases $\mu_{\max }=a$ instead of $\mu_{\max }=0$ and hence $\left.Q\right|_{S}$ is semistable but not stable.

\subsection{Congruences of type 17)}

Because of its difficulty, we study this case separately from the rest of the rational congruences (we will take this case as a model for the study of the other rational congruences). Recall that the congruence $S$ of this type is the blow-up of $\mathbb{P}^{2}$ in seven points, with hyperplane section $H=6 L-2 E_{1}-\cdots-2 E_{7}$.

Observe first that, for a general choice of seven points in $\mathbb{P}^{2}$, the linear system $\left|6 L-2 E_{1}-\cdots-2 E_{7}\right|$ defines an embedding of the blow-up of $\mathbb{P}^{2}$ into a smooth surface $S^{\prime} \subset \mathbb{P}^{6}$. Hence, it will produce a congruence if and only if $S^{\prime}$ is contained in a quadratic cone $Q^{\prime}$ with only one singular point $p^{\prime}$ not contained in the secant variety of $S^{\prime}$ (i.e. the linear projection from $p^{\prime}$ produces a smooth surface in $\mathbb{P}^{5}$ contained in a smooth quadric). We prove something stronger:

Claim 1. For a general choice of seven points in $\mathbb{P}^{2}$, there is a five-dimensional irreducible set $\Sigma$ of the linear system of quadrics containing $S^{\prime}$ such that $S^{\prime}$ is the intersection of all the quadrics of $\Sigma$ and the general quadric in $\Sigma$ has exactly one singular point, not lying in the secant variety of $S^{\prime}$.

Proof. Indeed, since there is a basis of $H^{0}\left(6 L-2 E_{1}-\cdots-2 E_{7}\right)$ of the form $s_{0}^{2}, s_{0} s_{1}, s_{0} s_{2}, s_{1}^{2}, s_{1} s_{2}, s_{2}^{2}, s$, where $s_{0}, s_{1}, s_{2}$ is a basis of $H^{0}\left(3 L-E_{1}-\cdots-E_{7}\right)$, then $S^{\prime}$ is contained in the cone $C_{p} V$ with vertex $p$ over a Veronese surface $V$. In particular, $S^{\prime}$ is contained in the five-dimensional family of quadrics containing $S^{\prime}$ (all of them singular at $p$ ). Moreover, it must be $h^{0}\left(\mathcal{J}_{S^{\prime}, \mathbb{P}^{6}}(2)\right)=7$ and $S^{\prime}$ must be the complete intersection of $C_{p} V$ with a quadric hypersurface in $\mathbb{P}^{6}$. Reciprocally, a general such intersection is isomorphic to the blow-up of $\mathbb{P}^{2}$ in seven points, with hyperplane section $H=6 L-2 E_{1}-\cdots-2 E_{7}$. Hence, it is enough to prove the claim for a general intersection of $C_{p} V$ with a quadric.

It follows from the description in [3] that a congruence of type 17) is contained only in one quadric of $\mathbb{P}^{5}$ (the Grassmannian), and it is the projection from $\mathbb{P}^{6}$ of a surface which is the complete intersection of a cone over the Veronese surface and a quadric hypersurface. This produces an example of $S^{\prime} \subset \mathbb{P}^{6}$ satisfying that the union of the singular points of the quadrics containing $S^{\prime}$ is not contained neither in the union of the singular lines of the quadrics containing $S^{\prime}$ nor in the secant variety of $S^{\prime}$ (observe that this secant variety must coincide with the secant variety of $C_{p} V$ ). Since this is an open condition, the general $S^{\prime}$ will also satisfy this property, hence it is contained in a quadratic cone whose singular locus is a point not contained in the secant variety of $S^{\prime}$.

We fix now a general $S^{\prime}$ as above and a general quadric cone $Q^{\prime}$ containing $S^{\prime}$ whose only singular point $p^{\prime}$ is not contained in the secant variety of $S^{\prime}$ (in 
particular, $Q^{\prime}$ would be the only quadric having $p^{\prime}$ in its singular locus). Then, in the six-dimensional linear system of the quadrics of $\mathbb{P}^{6}$ containing $S^{\prime}$, the set of singular quadrics forms a hypersurface of degree seven. It is not irreducible (since it contains at least one component corresponding to the quadrics containing $C_{p} V$ ). We take an irreducible component $\Sigma$ of this set containing the element $Q^{\prime}$. The claim will follow if we prove that $\Sigma$ is not a linear space.

Assume for contradiction that $\Sigma$ is linear. Then $\Sigma$ is contained in the tangent space at $Q^{\prime}$ of the set of singular quadrics in $\mathbb{P}^{6}$, i.e. the space of quadrics passing through $p^{\prime}$. But this is a contradiction, because $Q^{\prime}$ is the only singular quadric containing $S^{\prime}$ passing through $p^{\prime}$.

Since $S$ has no fundamental curve, we deduce from Theorem 2.1 that $D H \leq 3$ for all subsheaf $\mathcal{O}_{S}(D)$ of $\left.Q\right|_{S}$, hence $\left.Q\right|_{S}$ is stable. Now in order to compute the highest slope for $S$, observe first that there is no linear subsheaf of $\left.Q\right|_{S}$ with odd slope, since $D H$ is always even. We will show that, if $S$ is general, then there is no linear subsheaf of $\left.Q\right|_{S}$ with slope equal to 2 (hence the highest slope will be 0 ).

Claim 2. If $\mathcal{O}_{S}(D)$ is a subsheaf of $\left.Q\right|_{S}$ with slope equal to 2, then $D$ is one of the following:

i) $D=E_{i}$, with $i=1, \ldots, 7$,

ii) $D=L-E_{i_{1}}-E_{i_{2}}$, with $1 \leq i_{1}<i_{2} \leq 7$,

iii) $D=2 L-E_{i_{1}}-\cdots-E_{i_{5}}$, with $1 \leq i_{1}<\cdots<i_{5} \leq 7$

iv) $D=3 L-2 E_{i_{1}}-E_{i_{2}}-\cdots-E_{i_{7}}$, with $1 \leq i_{1} \leq 7,1 \leq i_{2}<\cdots<i_{7} \leq 7$, and $i_{1} \neq i_{j}$ for each $j=2, \ldots, 7$.

Proof. Suppose that $\mathcal{O}_{S}(D)$, with $D=d L-n_{1} E_{1}-\cdots-n_{7} E_{7}$, is a subsheaf of $\left.Q\right|_{S}$ with slope equal to 2 . We have thus a sequence like in (1.2), and the conditions $D H=2$ and $\operatorname{deg} Z \geq 0$ are equivalent to

$$
\begin{aligned}
& n_{1}+\cdots+n_{7}=3 d-1 \\
& n_{1}^{2}+\cdots+n_{7}^{2} \leq 2+d^{2} .
\end{aligned}
$$

From this, using the Cauchy-Schwarz inequality, we obtain

$$
2 d^{2}-6 d-13 \leq 0 .
$$

Hence the only possible values for $d$ are $-1,0,1,2,3$ or 4 . For shortness, we give the details only for the cases $d=-1,0$.

- If $d=-1$, we get the conditions

$$
\begin{gathered}
n_{1}+\cdots+n_{7}=-4 \\
n_{1}^{2}+\cdots+n_{7}^{2} \leq 3,
\end{gathered}
$$

which have no solution. 
- Suppose $d=0$. The solutions to

$$
\begin{gathered}
n_{1}+\cdots+n_{7}=-1 \\
n_{1}^{2}+\cdots+n_{7}^{2} \leq 2
\end{gathered}
$$

are all the divisors $D=E_{i}$, with $i=1, \ldots, 7$.

The divisors of ii) are obtained when $d=1$, whereas we get the divisors of iii) and iv) with the values $d=2$ and $d=3$, respectively. The value $d=4$ leads to no solution.

For a general choice of $S^{\prime}$ (hence a general choice of point $x_{1}, \ldots, x_{7}$ ) there is exactly one effective divisor in one of the above classes, yielding a total of 56 conics. Neither of these conics will provide a subsheaf of $\left.Q\right|_{S}$ if the plane they lie in is not a $\beta$-plane. We conclude the study of this case by proving the following:

Claim 3. Let $D_{1}^{\prime}, \ldots, D_{56}^{\prime} \subset S^{\prime}$ be the conics in the classes given in Claim 2 . Then the linear projection of $S^{\prime}$ from the vertex of a general $Q^{\prime} \in \Sigma$ produces a congruence such that the images of $D_{1}^{\prime}, \ldots, D_{56}^{\prime}$ are not contained in $\beta$-planes.

Proof. For each $i=1, \ldots, 56$, let $\Pi_{i}^{\prime}$ be the plane containing $D_{i}^{\prime}$ and let $p_{i}{ }^{\prime}$ a point of $\Pi_{i}^{\prime}$ outside $S^{\prime}$. Since $S^{\prime}$ is cut out by the quadrics of $\Sigma$, the general quadric in $\Sigma$ does not contain any $p_{i}{ }^{\prime}$, so that projecting from its vertex we get a surface in a smooth quadric in $\mathbb{P}^{5}$ (the Grassmannian) such that the image of any plane $\Pi_{i}^{\prime}$ is not contained in that quadric.

As a consequence, the highest slope of $\left.Q\right|_{S}$ is 0 if $S$ is a general congruence of this type.

\subsection{Rational congruences}

We start with a technical lemma, which shows that, under some conditions, a general rational congruence is the blow-up of $\mathbb{P}^{2}$ at points in general position:

Lemma 3.4. Let $\mathcal{H}$ be an irreducible component of the Hilbert scheme of congruences such that the general congruence $S$ in $\mathcal{H}$ is the blow-up of $\mathbb{P}^{2}$ in $r$ points, with hyperplane section $H_{S}=d L-n_{1} E_{1}-\cdots-n_{r} E_{r}$ and $h^{1}\left(\mathcal{O}_{S}(1)\right)=h^{1}\left(\mathcal{O}_{S}(2)\right)=$ 0. Moreover, suppose that:

(i) $\left(\begin{array}{c}d+2 \\ 2\end{array}\right)-\left(\begin{array}{c}n_{1}+1 \\ 2\end{array}\right)-\cdots-\left(\begin{array}{c}n_{r}+1 \\ 2\end{array}\right)=6$,

(ii) $1+2 d^{2}-2 n_{1}^{2}-\cdots-2 n_{r}^{2}+3 d-n_{1}-\cdots-n_{r} \leq 20$.

Then the general element of $\mathcal{H}$ is the blow-up of $\mathbb{P}^{2}$ at $r$ points in general position. 
Proof. Consider the blow-up $S^{\prime}$ of $\mathbb{P}^{2}$ at $r$ general points. From the existence of a congruence satisfying the open conditions of the statement, it follows $h^{1}\left(\mathcal{O}_{S^{\prime}}(1)\right)=$ 0 , so that (since $h^{2}\left(\mathcal{O}_{S^{\prime}}(1)\right)=0$ by Serre's duality) Riemann-Roch theorem and condition i) imply that $\left|d L-n_{1} E_{1}-\cdots-n_{r} E_{r}\right|$ has dimension five. Moreover, such a linear system gives an embedding in $\mathbb{P}^{5}$, because it does for the congruences in $\mathcal{H}$. In the same way, conditions $h^{1}\left(\mathcal{O}_{S^{\prime}}(2)\right)=0$ and (ii) imply that the image of $S^{\prime}$ in $\mathbb{P}^{5}$ lies on a quadric (which is smooth in general, because of the existence of congruences in $\mathcal{H})$. Finally, identifying such a smooth quadric with $\mathbb{G}(1,3)$, we get that the general $S^{\prime}$ determines a congruence, as wanted.

We compute now the highest slope for the different types of rational congruences. In most of the cases we will just sketch the proof (the complete details can be found in [5]).

Case 6) In this case, $S=\widetilde{\mathbb{P}^{2}}\left(x_{1}, \ldots, x_{5}\right)$ with hyperplane section $H=3 L-E_{1}-$ $\cdots-E_{5}$ and the congruences of this type are degenerate, i.e. contained in a linear complex.

If this linear complex is general, then $\left.Q\right|_{S}$ is stable by Theorem 2.1 and the highest slope 1 is reached by every $\mathcal{O}_{S}\left(E_{i}\right)$. On the contrary, if the linear complex is special, then there exists a linear subsheaf of $\left.Q\right|_{S}$ with slope equal to 2 (see Theorem 2.1). Hence $\left.Q\right|_{S}$ is semistable, but not stable, and the highest slope is 2 .

Case 7) Theorem 2.1 yields $\mu_{\max } \leq 1$, and this value is attained for any $D=E_{i}$, with $i=1, \ldots, 4$.

Case 7)* Now Theorem 2.1 yields $\mu_{\max } \leq 2$. Considering the dual congruence, [1], Example 1.14 shows that the pencils of conics $\left|L-E_{i}\right|$ or $\left|2 L-E_{1}-\cdots-E_{5}\right|$, always contain some conic lying in a $\beta$-plane. Hence, any such conic reaches the highest slope 2 .

Case 10) By Theorem 2.1, $\mu_{\max } \leq 2$, and this is achieved by any $D=L-E_{i}$.

Case 11). Theorem 2.1 yields $\mu_{\max } \leq 2$ and that, for any subsheaf of $\left.\mathcal{O}_{S}(D) \subset Q\right|_{S}$ of slope two, it follows that $D$ is effective, i.e. a conic in a $\beta$-plane. Proceeding as in Claim 2 of case 17), the only possibilities for $D$ are

$$
\begin{aligned}
D= & L-E_{1} \\
D= & E_{1} \text { or } D=3 L-2 E_{1}-E_{2}-\cdots-E_{7} \\
D= & L-E_{i}-E_{j} \text { or } D=2 L-E_{1}-E_{2}-\cdots-E_{7}+E_{i}+E_{j}, \\
& \text { with } i, j=2, \ldots, 7 .
\end{aligned}
$$

Observe that $\mathcal{O}_{S}\left(L-E_{1}\right)$ cannot be a subsheaf of $\left.Q\right|_{S}$, since otherwise any conic in $\left|L-E_{1}\right|$ would lie on a $\beta$-plane, implying that the dual congruence would have a fundamental curve. Hence we have only 32 possible conics $C_{1}, \ldots, C_{32}$ to lie on a $\beta$-plane. However, since the congruence, as a surface $S \subset \mathbb{P}^{5}$ is cut-out by quadrics, the general quadric containing $S$ will not contain any of planes $<C_{1}>$ $, \ldots,<C_{32}>$ (for each $i=1, \ldots, 32$, choose a point $p_{i}$ in $<C_{i}>\backslash S$ and pick 
a quadric avoiding $p_{1}, \ldots, p_{32}$ ). Such a general quadric will be smooth (because $S$ is contained in a smooth quadric), so that we can identify it with $\mathbb{G}(1,3)$. Therefore the general congruence of this type contains no conic lying in a $\beta$-plane.

Hence $\mu_{\max }=1$ for a general congruence of this type (for example $D=E_{2}$ achieves this value). However, special types of this congruence can have one, two or three conics lying in a $\beta$-plane ( [1, Example 1.15]) and in these cases $\mu_{\max }=2$.

Case 13)* By Theorem 2.1, $\mu_{\max } \leq 3$. This bound is achieved $D=3 L-E_{1}-\cdots-$ $E_{9}$. Indeed, $D$ is a plane cubic, hence it is contained in an $\alpha$-plane or $\beta$-plane (see Remark 1.8). It is not contained in an $\alpha$-plane, since it would produce a subsheaf of slope three for the dual congruence, in contradiction with Theorem 2.1.

Case 13) By Theorem 2.1, $\mu_{\max } \leq 2$, and $\mu(D)=2$ only if $D$ is effective. We exclude that possibility by a computation as in case 17), Claim 2, with some extra difficulties that we specify only in this case:

- The possibility $D=E_{i}+E_{j}$ is excluded because $E_{i}, E_{j}$ cannot lie on the same $\beta$-plane, since they do not meet.

- The conic $D=L-E_{i}-E_{j}$ is not contained in a $\beta$-plane because it meets the cubic $C=3 L-E_{1}-\cdots-E_{9}$ (which is in an $\alpha$-plane, as we have seen when studying the dual congruence) in one point and $C \cup D$ span a hyperplane in $\mathbb{P}^{5}$.

- We need to use Lemma 3.4 to exclude cases like $D=2 L-E_{1}-\cdots-E_{6}$, which are not effective for a general $S$.

Finally, since any $D=E_{i}$ has slope one, $\mu_{\max }=1$ if the congruence is general.

Cases 16) Now $\mu_{\max } \leq 2$, by Theorem 2.1, and slope two can be reached only by effective divisors. This is excluded for a general congruence with the help of Lemma 3.4, while slope one is achieved for any $D=E_{i}$.

Case 16)* Theorem 2.1 shows $\mu_{\max } \leq 4$, which is excluded by the usual techniques, while the slope 3 is reached by $\mathcal{O}_{S}\left(3 L-E_{1}-\cdots-E_{9}\right)$.

Case 18) In this case, Theorem 2.1 provides $\mu_{\max } \leq 3$, and this is achieved only for effective divisors $D$, which is excluded by Lemma 3.4. Since $D=3 L-E_{1}-$ $\cdots-E_{11}$, is in a $\beta$-plane (because $h^{0}\left(\left.Q\right|_{S}(-D)\right)>0$ by Riemann-Roch), it follows $\mu_{\max }=2$ if $S$ is general.

Case 21) Theorem 2.1 shows now $\mu_{\max } \leq 2$, and for a general congruence we exclude the possibilities $\mu_{\max }=1,2$ as usual, using Lemma 3.4. Hence $\mu_{\max }=0$ if $S$ is general.

Case 21)* In this case, $\mu_{\max } \leq 5$ by Theorem 2.1 , and we exclude the possibilities $\mu_{\max }=4,5$ by the usual Cauchy-Schwarz trick. The slope three is reached by $\mathcal{O}_{S}\left(3 L-E_{1}-\cdots-E_{9}\right)$, so that $\mu_{\max }=3$ for any congruence of this type.

Case 22) Now we get here $\mu_{\max } \leq 3$ from Theorem 2.1, and we exclude the cases $\mu_{\max }=2,3$ with the help of Lemma 3.4. Then $\mu_{\max }=1$ if $S$ is general, and this is achieved by any $D=E_{i}$. 
Case 22)* Finally, Theorem 2.1 yields $\mu_{\max } \leq 4$. We exclude, as usual the cases $\mu_{\max }=3,4$ (we need now to use Lemma 3.4 to exclude possibilities like $D=$ $3 L-E_{1}-\cdots-E_{10}$, because then $h^{0}(D)=0$ and $h^{0}(H-D)=3$, which is incompatible with the existence of an exact sequence like (1.2)). Hence $\mu_{\max }=2$ if $S$ is general, and this value is achieved by $D=3 L-E_{1}-\cdots-E_{11}$.

\subsection{Conic bundles}

Case 15). By Theorem 2.1, $\mu(D) \leq 2$ and $\mu(D) \neq 1$ for all subsheaf $\mathcal{O}_{S}(D)$ of $\left.Q\right|_{s}$. We will prove $\mu_{\max }=0$ by excluding the case $\mu(D)=2$ as in the case of rational congruences, but now writing numerical equivalence $D \equiv p C_{0}+q f$, with $p, q \in \mathbb{Z}$. Then $D H=2$ yields $3 p+2 q=2$, hence $D \equiv-2 t C_{0}+(1+3 t) f$, for some $t \in \mathbb{Z}$, while $c_{2}\left(\left.Q\right|_{S}(-D)\right) \geq 0$ yieds $(-4)\left(t-\frac{1}{2}\right)(t+1) \geq 0$, so that $t=-1$ or $t=0$. Let us exclude both possibilities:

- If $t=0$, then $D$ is a fiber of the elliptic surface, embedded as a conic, which is necessarily contained in a $\beta$-plane. This is impossible, because the congruence is the set of bisecant lines of an elliptic quartic curve in $\mathbb{P}^{3}$, so that every plane of $\mathbb{P}^{3}$ contains at most six lines of the congruence.

- If $t=-1$, then $D \equiv 2 C_{0}-2 f$ and $c_{2}\left(\left.Q\right|_{S}(-D)\right)=0$, so that we have an exact sequence

$$
\left.0 \longrightarrow \mathcal{O}_{S}\left(2 C_{0}-2 f\right) \longrightarrow Q\right|_{S} \longrightarrow \mathcal{O}_{S}(H-D) \longrightarrow 0
$$

with $H-D \equiv 3 f$. Hence $h^{0}(H-D)=3$ and therefore $D$ is effective, i.e. a conic contained in a $\beta$-plane, which is impossible as in case $t=0$.

Case 15)*. In this case, $\mu_{\max } \leq 4$ by Theorem 2.1. Arguing as in the above case, we exclude the existence of a subsheaf $\mathcal{O}_{S}(D)$ with $\mu(D)=4$, since it should be $D \equiv-2 t C_{0}+(2+3 t) f$, for some $t \in \mathbb{Z}$, while $c_{2}\left(\left.Q\right|_{S}(-D)\right)=-8 t^{2}-8 t-2 \geq 0$ gives no solution. It is $\mu_{\max }=3$, since this is achieved by $D=C_{0}$. Indeed, $C_{0}$ is a plane cubic, hence contained in an $\alpha$-plane or a $\beta$-plane (see Remark 1.8). It cannot be contained in an $\alpha$-plane, since this would yield $\mu_{\max } \geq 3$ for the dual congruence, while we proved it is zero.

Case 25). Recall from [2], Remark 3.6 that a congruence of this type can have a fundamental curve (namely a smooth plane cubic), so that $\mu_{\max }=3$ by Proposition 3.1, while the general one has no fundamental curve, so that Theorem 2.1 implies $\mu_{\max } \leq 1$. We have equality, because the conic bundle has three singular conics, hence $S$ contains six lines.

Case 25)*. The main difficulty of this case is that we do not know the precise value of $e$ (at least for the general congruence). However, using that $C_{0}$ is elliptic, hence of degree at least three, we get $e \leq 0$. On the other hand, since $e \geq-1$ (see [11]) we get $e=-1,0$. Now we can proceed as in the case of rational congruences: 
Applying now Theorem 2.1, we get that $D H \leq 4$ for all $\left.\mathcal{O}_{S}(D) \subset Q\right|_{S}$, with equality only if $D$ is effective, hence $D$ would be a quartic curve contained in a $\beta$ plane. To prove that this is impossible, write $D \equiv p C_{0}+q f-n_{1} E_{1}-\cdots-n_{r} E_{r}$. The conditions $D H=4$ and $c_{2}\left(\left.Q\right|_{S}(-D)\right) \geq 0$ yield

$$
\begin{aligned}
n_{1}+n_{2}+n_{3} & =(3-e) p+2 q-4 \\
n_{1}^{2}+n_{2}^{2}+n_{3}^{2} & \leq-1-p^{2} e+2 p q
\end{aligned}
$$

and applying to them the Cauchy-Schwarz inequality we obtain

$$
\left(e^{2}-3 e+9\right) p^{2}+[2 q(3-2 e)-8(3-e)] p+4 q^{2}-16 q+19 \leq 0 .
$$

From this we get the solutions

$$
D \equiv C_{0}+f-E_{i} \quad \text { or } \quad D \equiv C_{0}+2 f-E_{1}-E_{2}-E_{3} \quad \text { if } \quad e=0
$$

(excluded because they have arithmetical genus equal to one, so that they cannot be plane quartics) and

$$
D \equiv C_{0} \quad \text { or } \quad D \equiv C_{0}+f-E_{i}-E_{j} \quad \text { if } \quad e=-1
$$

(excluded since they have arithmetical genus zero).

We finally show $\mu_{\max }=3$ by showing a cubic lying in a $\beta$-plane for each values of $e$. Since the dual congruence has $\mu_{\max }=2$, this plane cubic will be necessarily in a $\beta$-plane. If $e=0$, we take $D=C_{0}$. If instead $e=-1$, there exist infinitely many choices for $C_{0}$, so that we can choose one passing through the point $p_{1}$ we are blowing up. We take then the effective divisor $D=C_{0}-E_{1}$, which has degree three and arithmetical genus one, hence it is a plane cubic.

\section{Appendix. Congruences of degree 10}

We include here, in Table 3.1 and without proof, a partial result of the stability of $\left.Q\right|_{S}$ for nondegenerate congruences of degree 10 (for the degenerate case, see Remark 3.3). These congruences were classified by Gross in [9], although the irreducibility of the corresponding Hilbert schemes is not proved. Hence the possible values of $\mu_{\max }$ that we obtained have not full meaning (anyway, $\left.Q\right|_{S}$ is always stable by Theorem 2.1). In the last column of the table we write down the divisor $D$ such that $\mu(D)$ is the highest possible slope we have found. For example, the options we give for the congruence $(4,6)$ blow-up of a $K 3$ surface and its dual mean that $\mu_{\max }=2$ if and only if $\mathcal{O}_{S}(E)$ is a subsheaf of $\left.Q\right|_{S}$; otherwise $\mu_{\max }=0$, and it is reached by the subsheaf $\mathcal{O}_{S}$. 


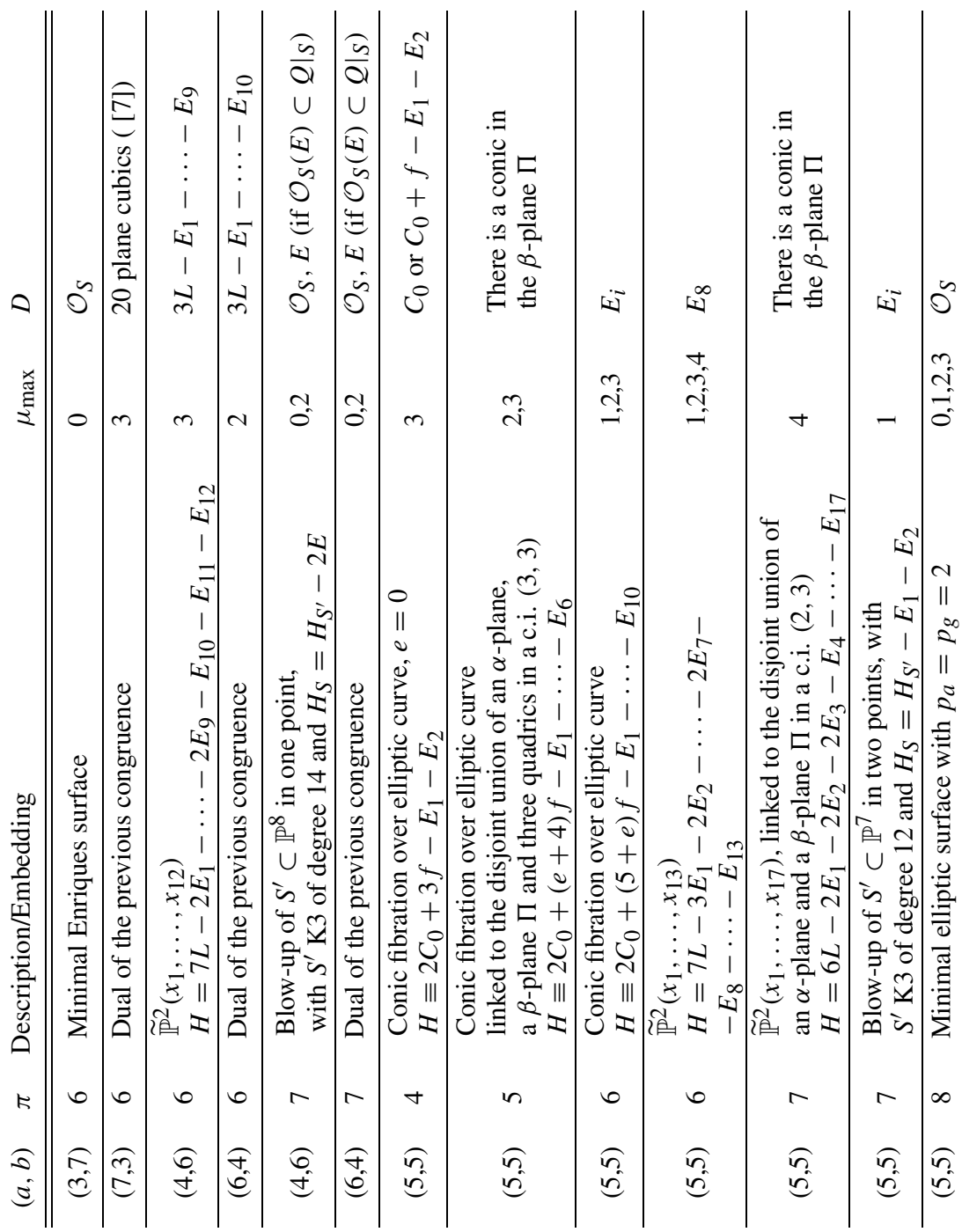

Table 3.1. Stability properties for congruences of degree 10 .

\section{References}

[1] E. Arrondo, M.Bertolini and C. TURrini, A focus on focal surfaces, Asian J. Math. 3 (2001), 535-560.

[2] E. ARrondo and M. GRoss, On smooth surfaces in $G\left(1, \mathbf{P}^{3}\right)$ with a fundamental curve, Manuscripta Math. 79 (1993), 283-298. 
[3] E. Arrondo and I. Sols, "On Congruences of Lines in the Projective Space”, Mém. Soc. Math. France, Vol. 50, 1992.

[4] S. Сово, Simplicity of the universal quotient bundle restricted to congruences of lines in $\mathbb{P}^{3}$, Adv. Geom. 6 (2006), 467-473.

[5] S. Сово, "Estabilidad del Fibrado Universal Restringido a Congruencias", PhD Thesis, Universidad Complutense de Madrid, 2008.

[6] P. Deligne and N. Katz, "Groupes de Monodromie en Géométrie Algébrique", SGA7II, Springer LNM 340, 1973.

[7] I. DolgacheV and I. ReIDER, On rank 2 vector bundles with $c_{1}^{2}=10$ and $c_{2}=3$ on Enriques surfaces, In: "Algebraic Geometry" (Chicago, IL), Lecture notes in Mahtematics, Springer-Verlag, Vol. 1479, 1991.

[8] M. GRoss, The distribution of bidegrees of smooth surfaces in $\mathbb{G}(1,3)$, Math. Ann. 292 (1992), 127-147.

[9] M. GRoss, Surfaces of degree 10 in the Grassmannian of lines in 3-space, J. Reine Angew. Math. 436 (1993), 87-127.

[10] R. HARTShorne, "Algebraic Geometry”, Springer, 1997.

[11] M. NAGATA, On self-intersection number of a section on a ruled surface, Nagoya Math. J. 37 (1970), 191-196.

Departamento de Álgebra

Facultad de Ciencias Matemáticas

Universidad Complutense de Madrid

28040 Madrid, Spain

arrondo@mat.ucm.es

scobopab@mat.ucm.es 